unavoidable. The positive identification of the aeroallergen that is responsible for allergic rhinitis symptoms is of great importance for choosing the right therapeutic approach.

\section{PO-1029 DIAGNOSTIC VALUE OF APOPTOTIC ACTIVITY AND OXIDATIVE STRESS IN COMMUNITY-ACQUIRED PNEUMONIA IN INFANTS}

H Pavlyshyn, I Sarapuk, V Slyva, N Haliyash. Pediatrics, I. Ya. Horbachevsky Ternopil State Medical University, Ternopil, Ukraine

\subsection{6/archdischild-2014-307384.1645}

Background Community-acquired pneumonia is one of the most studied pathologies of childhood, while the problem of diagnostics the disease severity still remains.

Aim To increase the effectiveness of diagnostics the severity of community-acquired pneumonia in infants by studying the processes of neutrophils' apoptotic activity and oxidative stress.

Methods The study involved 73 infants with communityacquired pneumonia (moderate severity of disease was diagnosed in 44 children (I group); severe pneumonia - in 29 (II group)) and 23 healthy children of the control group. The intensity of neutrophils' apoptosis and necrosis was determined by flow cytometry, the generation of superoxide anion $\left(\mathrm{O}_{2}^{-}\right)$- by spectrophotometric method, reactive oxygen species (ROS) - by fluorometric method.

Results The intensive growth of neutrophils' apoptosis was revealed in infants with community-acquired pneumonia of moderate severity $\left(\mathrm{p}_{\mathrm{c}-1}<0.001\right)$, while necrosis prevailed in severe pneumonia $\left(\mathrm{p}_{\mathrm{c}-2}<0.001\right)$.

The oxidative stress indicators correlated with disease severity. ROS generation indices in infants of I group - 17.23 (16.29; 18.73) RFU·ml ${ }^{-1}$, II group - $24.11(21.23 ; 25.75) \mathrm{RFU} \cdot \mathrm{ml}^{-1}, \mathrm{p}_{\mathrm{c}-1}$ $<0.001, \mathrm{p}_{\mathrm{c}-2}<0.001 ; \mathrm{O}_{2}^{-}-1.13(1.06 ; 1.14) \mathrm{nmol} \cdot(\mathrm{min} \cdot \mathrm{mL})^{-1}$ and $1.41(1.33 ; 1.49) \mathrm{nmol} \cdot\left(\mathrm{min} \cdot \mathrm{mL}^{-1}\right.$ accordingly, $\mathrm{p}_{\mathrm{c}-1}<0.001$, $\mathrm{p}_{\mathrm{c}-2}<0.001$.

The method of multiple regression analysis showed, that lowering of saturation in infants with severe pneumonia for $33.8 \%$ is interrelated with the changes of neutrophils' apoptosis and necrosis, determined by the determination index, and confirm the diagnostic value of apoptotic activity.

Conclusion The oxidative stress indicators allow to determine the community-acquired pneumonia severity in infants, and intensification of neutrophils' necrosis with simultaneous inhibition of apoptotic activity is an accurate criterion of severe pneumonia.

\section{PO-1030 HEMOPTYSIS - THE ALARMING SYMPTOM IN PAEDIATRIC CARE - FOUR DIFFERENT CASES}

${ }^{1} \underline{G}$ Petrova, ${ }^{1} D$ Miteva, ${ }^{1} \mathrm{P}$ Perenovska, ${ }^{1} \mathrm{R}$ Kabakchieva, ${ }^{2} \mathrm{O}$ Brankov. ${ }^{1}$ Pediatric Clinic, University Hospital "Alexandrovska", Sofia, Bulgaria; ${ }^{2}$ Pediatric Surgery Department, Medical University Sofia, Sofia, Bulgaria

\subsection{6/archdischild-2014-307384.1646}

Hemoptysis is a symptom in a various diseases with different causes and pathology. In some cases blood-laced mucus from the sinus or nose can sometimes be misidentified as symptomatic of hemoptysis is a minor problem, but in others with true hemoptysis the condition could be life-threatening. We present 4 different cases of hemoptysis.
Case1: 15-year-old girl with cystic fibrosis, with a surgical resection of her lower left lobe due to gangrene previous year, presented with severe hemoptysis. She underwent bronchoscopy followed by thoracotomy and resection of right upper lobe.

Case2: 9-year-old boy with chronic cough with abundant greenish, smelly sputum, with blood stains, easy fatigue, dyspnea at exercises, recurrent pneumonia since early childhood. The boy is cachectic with short stature, asymmetrical rib cage, genu varum and bilateral ptosis. CAT confirmed bronchiectasis.

Case 3: 17-year-old girl with hazardous behaviour admitted in the clinic for hemoptysis and severe changes on the X-ray of the lungs. Two weeks after admission and a week after initiation of corticosteroid therapy for suspected hemosiderosis a severe hematuria occurred. Goodpasture's syndrome was confirmed and the therapy was modified.

Case: 15-year-old boy admitted for "epistaxis" and X-ray changes with possible pneumonia. Varices of nasal septum was confirmed, but true hemoptysis was present due to hydatid cyst in the left lung.

Conclusion In presence of the alarming sign of hemoptysis, a full differential follow-up should be performed for not neglecting severe conditions that could be life-threatening.

\section{PO-1031 FENO AND ATOPIC STATUS IN CHILDREN}

${ }^{1}$ DA Plesca, ${ }^{1}$ E Buzoianu, ${ }^{2} \mathrm{M}$ Moiceanu, ${ }^{1} \mathrm{VS}$ Plesca, ${ }^{2} \mathrm{~L}$ Spatariu, ${ }^{1} \mathrm{~F}$ Cora. ${ }^{1}$ Pediatric and Pediatric Neurology Children Clinical Hospital "Victor Gomoiu", UMF Carol Davila, Bucharest, Romania; ${ }^{2}$ Pediatric Department, Children Clinical Hospital "Victor Gomoiu", Bucharest, Romania

\subsection{6/archdischild-2014-307384.1647}

Background and aim FeNO (fractional exhaled NO) is an useful tool to assess asthma control, being correlated with eosinophilic inflammation of the respiratory tract. Nevertheless FeNO value varies according to asthma control status in just one subgroup of patients.

The aim of this study is to determine if atopy represent a feature that define the subgroup of patients for which FeNO is an appropriate tool for disease control assessment.

Method We have conducted a retrospective study including 47 patients diagnosed with bronchial asthma in our clinic between January-December 2013. From this group we have counted those patients who had during this period at least one testing with $\mathrm{FeNO}$ value $>25 \mathrm{ppb}$ (increased above normal limit and related to poorly controlled asthma); the variation of FeNO values is assigned to changes in asthma control status. Also from the same group we have spotted those patients who had atopy. Finally we have correlated the 2 sungroups: those with at least one FeNO value $>25 \mathrm{ppb}$ and those with atopy using Pearson Chi-Square test.

Results From 35 patient with atopy 18 patients had at least one testing with FeNO $>25$ ppb (51,4\%); from 12 patients without atopy only one patient had at least one testing with FeNO $>25$ ppb (8,3\%); Pearson Chi-Square test estimated that FeNO-atopy correlation is statistically significant $(\mathrm{p}=0,013)$.

Conclusion FeNO value is correlated with atopy in asthmatic patients and atopy can be considered a feature that define the subgroup of asthmatic patients eligible to be monitored using FeNO. 